\title{
6-dimensional brane world model
}

\author{
Panagiota Kanti \\ Scuola Normale Superiore, Piazza dei Cavalieri 7, I-56126 Pisa, Italy \\ Richard Madden \\ Institut des Hautes Études Scientifiques, 91440 Bures-sur-Yvette, France
}

Keith A. Olive

Theoretical Physics Institute, School of Physics and Astronomy, University of Minnesota, Minneapolis, Minnesota 55455

(Received 24 April 2001; published 27 July 2001)

\begin{abstract}
We consider a 6-dimensional spacetime which is periodic in one of the extra dimensions and compact in the other. The periodic direction is defined by two 4-brane boundaries. Both static and nonstatic exact solutions, in which the internal spacetime has a constant radius of curvature, are derived. In the case of static solutions, the brane tensions must be tuned as in the 5-dimensional Randall-Sundrum model; however, no additional finetuning is necessary between the brane tensions and the bulk cosmological constant. By further relaxing the sole fine-tuning of the model, we derive nonstatic solutions, describing de Sitter or anti-de Sitter 4-dimensional spacetimes, that allow for the fixing of the interbrane distance and the accommodation of pairs of positivenegative and positive-positive tension branes. Finally, we consider the stability of the radion field in these configurations by employing small, time-dependent perturbations around the background solutions. In analogy with results drawn in five dimensions, the solutions describing a de Sitter 4-dimensional spacetime turn out to be unstable while those describing an anti-de Sitter geometry are shown to be stable.
\end{abstract}

DOI: 10.1103/PhysRevD.64.044021 PACS number(s): 04.50.+h, 11.25.Mj, 11.27.+d, 98.80.Cq

\section{INTRODUCTION}

It would be an understatement to say that the possibility of resolving the hierarchy problem in models with a warped extra dimension [1] has received considerable attention over the last two years. Indeed, brane world models have dominated the literature in high energy theory. The reason lies in its simplicity. By postulating the existence of two 3-branes with nonzero tensions, separated along the extra dimension by a distance $L$, in the background of a nonzero (negative) cosmological constant, one finds a simple solution for the scale factor along the extra dimension, $a(y)$, which is exponential. Thus length scales (and hence mass scales) on one brane are exponentially enhanced (or suppressed) relative to the other. A mass hierarchy naturally arises between the two branes which can be labeled the Planck and weak branes, respectively.

Of course, there is a price to pay for this simplicity. First, as is well known, the tensions of the two 3-branes must be fine-tuned so that $\Lambda_{1}=-\Lambda_{2}$. Second, these tensions must be tuned to the bulk cosmological constant $\Lambda_{B}$ in order to produce a static solution. The origins of these fine-tunings come about when one considers static solutions to the 5 -dimensional (5D) equations of motion. The scale factor in the extra direction takes the form $a(y)=e^{-k y}$ and the equations of motion require $k^{2}=-\kappa_{5}^{2} \Lambda_{B} / 6$, where $\kappa_{5}^{2}$ is the $5 \mathrm{D}$ Newton constant. We are, therefore, led to an anti-de Sitter 5D spacetime with $\Lambda_{B}<0$. By putting branes in the theory, and requiring that the warp factor exhibits periodic behavior along the extra dimension, we obtain the so-called jump conditions which give $\left[a^{\prime}(y)\right]_{i} / a_{i}=-\kappa_{5}^{2} \Lambda_{i} / 3$, where $\left[a^{\prime}\right]$ represents the difference in $a^{\prime}$ on the two sides of the brane. For one brane, say with positive tension placed at the origin $(y$
$=0)$, we see that $k=\kappa_{5}^{2} \Lambda_{1} / 6$, and for the second brane, located at an undetermined distance $L$, one finds $k=$ $-\kappa_{5}^{2} \Lambda_{2} / 6$. Thus we arrive at the conditions $\Lambda_{1}=-\Lambda_{2}$ $=\sqrt{6 \Lambda_{B} / \kappa_{5}^{2}}$. Finally, the distance $L$ is chosen so as to resolve the hierarchy problem by noting that masses scale as $a(y)$. For other recent attempts at solving the hierarchy problem with extra dimensions see $[2,3]$.

Several extra-dimensional attempts at resolving the hierarchy, or the cosmological constant, problem have considered six- or higher-dimensional models [4,5]. Spacetimes with more than one extra dimension can allow for solutions with the most appealing features, particularly in spacetimes where the curvature of the internal space is nonzero. These solutions, exhibiting either spherical or cylindrical symmetry with respect to the extra coordinates, can accommodate an exponential dependence on one of the extra coordinates, thus, resembling the 5D Randall-Sundrum (RS) mechanism for the resolution of the hierarchy problem. In addition, it turns out that such spacetimes can play an important role in relaxing the degree of fine-tuning in the RS models [5]. Finally, these models can provide a framework in the context of which the stabilization of the radion field naturally takes place: for example, in Ref. [6] it was shown that in spacetimes with a constant spatial curvature of the internal dimensions, one can find solutions with a global minimum in the effective theory for the radion field.

In this paper we look for solutions to the 6-dimensional (6D) equations of motion based on an internal space of constant curvature. We first present an exact static solution where the warp factor depends on both extra coordinates and, hence, does not exhibit any spherical or cylindrical symmetry. The dependence on one of the two extra coordinates is a purely exponential one thus resembling the profile of the 
warp factor in the case of the 5D RS model. In analogy with RS1, the spacetime contains two 4-brane boundaries with equal and opposite brane tensions. This configuration requires the same fine-tuning that exists in the RS1 model due to the jump conditions imposed at the boundaries. However, the solution does not contain the additional fine-tuning between the brane tensions and the bulk cosmological constant which is replaced by the fixing of the size of the extra dimension along which the 4-branes extend. The interbrane distance along the remaining extra dimension remains arbitrary and it may be fixed only through the introduction of an additional mechanism for the stabilization of the radion field $[7,8]$.

We then proceed to derive nonstatic solutions in the context of the same model. In this case, the exponential behavior along one of the two extra coordinates changes to cosh or sinh-like allowing for the accommodation of pairs of branes with positive tensions or positive-negative, respectively. The jump conditions lead to the fixing of the locations of the two branes along the same dimension and the fine-tuning between the brane tensions disappears rendering this solution totally free of any fine-tuning.

Both of the above solutions, static or nonstatic, have been derived under the assumption that the extra spacetime remains static. We formulate an "extremization" constraint that may serve as a consistency check for any 6D solution with a constant or nonconstant radion field. We finally perform a stability analysis around the solutions with a constant "extra" scale factor in order to check their stability under small time-dependent perturbations. We find, in agreement with similar results derived for 5D spacetimes [9-14], that the system of two Minkowski 4-dimensional (4D) subspaces has a vanishing radion mass, a pair of two de Sitter ones has a negative mass squared, while the system of two anti-de Sitter 4D subspaces has a positive mass squared.

In the next section we present the model and derive the exact static solution in Sec. III. We show explicitly how the correlation between the brane tensions and the bulk cosmological constant is replaced by the fixing of the size of one of the two extra dimensions. In Sec. IV we show how the relaxation of the fine-tuning between the brane tensions leads to de Sitter or anti-de Sitter expansions in the 4D spacetime in analogy with 5D models. The "extremization" constraint and the stability analysis of our solutions are discussed in Sec. V. Finally, we present our conclusions in Sec. VI.

\section{THE GENERAL FRAMEWORK}

Let us start by presenting the theoretical framework and geometrical setup of our model. We first write down the action that describes the gravitational theory of a 6-dimensional spacetime filled with a bulk cosmological constant as ${ }^{1}$

\footnotetext{
${ }^{1}$ Throughout this paper we follow Wald's conventions [15]: The metric signature is $\eta_{M N}=(-,+, \ldots,+)$ and the Riemann tensor is defined as $R_{\rho \mu \nu}^{\sigma}=\partial_{\mu} \Gamma_{\rho \nu}^{\sigma}-\partial_{\nu} \Gamma_{\rho \mu}^{\sigma}+\cdots$.
}

$$
S=-\int d^{6} x \sqrt{-G_{6}}\left\{-\frac{R_{(6)}}{2 \kappa_{6}^{2}}+\Lambda_{B}\right\},
$$

where $\kappa_{6}^{2}=8 \pi / M_{6}^{4}$. The line-element of the six-dimensional spacetime is assumed to be of the form

$$
d s^{2}=a^{2}(t, \theta, \varphi) \eta_{\mu \nu} d x^{\mu} d x^{\nu}+b^{2}(t)\left[d \theta^{2}+f^{2}(\theta) d \varphi^{2}\right] .
$$

In the above, $x^{\mu}$ and $(\theta, \phi)$ denote the coordinates along the usual four and two extra, all (initially) noncompact, dimensions, respectively. The function $a(t, \theta, \varphi)$ represents the warp factor multiplying the 4-dimensional line element, $b(t)$ denotes the scale factor that determines the dynamics of the 2-dimensional extra spacetime, while $f(\theta)$ parametrizes its internal curvature. For reasons that will become clear shortly, we will often refer to the $\theta$ dimension as the "longitudinal" one and to the $\varphi$ dimension as the "transverse" one.

In this paper we will focus on the determination of static and nonstatic solutions under the assumption that the scale factor along the extra dimensions remains always constant. As noted in the Introduction, we are not assuming any specific mechanism for radion stabilization which would generate additional stress-energy terms $[7,8]$. We will instead assume that the internal curvature of the 2-dimensional extra spacetime will naturally lead the system to solutions with a constant radion field that correspond to a minimum of the radion effective potential as in Ref. [6]. The outcome of this attempt is not, however, straightforward: in [6], it was assumed that $a$ and $b$ are functions of the coordinates $x^{\mu}$ only. None of the scale factors depend on the internal coordinates $\theta$ and $\phi$ and, thus, their results are not directly applicable to any warped brane model.

Upon variation with respect to the $6 \mathrm{D}$ metric tensor, the above action and metric ansatz leads to the following Einstein's equations in the bulk:

$$
\begin{aligned}
G_{00}= & \frac{\dot{b}^{2}}{b^{2}}+\frac{6 \dot{b} \dot{a}}{b a}+\frac{3 \dot{a}^{2}}{a^{2}}-\frac{a^{2}}{b^{2}} \frac{f^{\prime \prime}}{f}-\frac{3}{b^{2} f^{2}}\left(\frac{\partial a}{\partial \varphi}\right)^{2}-\frac{3 a}{b^{2} f^{2}} \frac{\partial^{2} a}{\partial \varphi^{2}} \\
& -\frac{3 a f^{\prime}}{b^{2} f} \frac{\partial a}{\partial \theta}-\frac{3}{b^{2}}\left(\frac{\partial a}{\partial \theta}\right)^{2}-\frac{3 a}{b^{2}} \frac{\partial^{2} a}{\partial \theta^{2}}=\kappa_{6}^{2} a^{2} \Lambda_{B}, \quad(2.3 \\
G_{i i}= & -\frac{\dot{b}^{2}}{b^{2}}-\frac{2 \ddot{b}}{b}-\frac{2 \dot{b} \dot{a}}{b a}+\frac{\dot{a}^{2}}{a^{2}}-\frac{2 \ddot{a}}{a}+\frac{a^{2}}{b^{2}} \frac{f^{\prime \prime}}{f}+\frac{3}{b^{2} f^{2}}\left(\frac{\partial a}{\partial \varphi}\right)^{2} \\
& +\frac{3 a}{b^{2} f^{2}} \frac{\partial^{2} a}{\partial \varphi^{2}}+\frac{3 a f^{\prime}}{b^{2} f} \frac{\partial a}{\partial \theta}+\frac{3}{b^{2}}\left(\frac{\partial a}{\partial \theta}\right)^{2}+\frac{3 a}{b^{2}} \frac{\partial^{2} a}{\partial \theta^{2}} \\
= & -\kappa_{6}^{2} a^{2} \Lambda_{B}, \\
G_{55}= & -\frac{b \ddot{b}}{a^{2}}-\frac{2 b \dot{b} \dot{a}}{a^{3}}-\frac{3 b^{2} \ddot{a}}{a^{3}}+\frac{6}{a^{2} f^{2}}\left(\frac{\partial a}{\partial \varphi}\right)^{2}+\frac{4}{a f^{2}} \frac{\partial^{2} a}{\partial \varphi^{2}} \\
& +\frac{4 f^{\prime}}{a f} \frac{\partial a}{\partial \theta}+\frac{6}{a^{2}}\left(\frac{\partial a}{\partial \theta}\right)^{2}=-\kappa_{6}^{2} b^{2} \Lambda_{B},
\end{aligned}
$$




$$
\begin{aligned}
G_{66}= & -\frac{b f^{2} \ddot{b}}{a^{2}}-\frac{2 b f^{2} \dot{b} \dot{a}}{a^{3}}-\frac{3 b^{2} f^{2} \ddot{a}}{a^{3}}+\frac{6}{a^{2}}\left(\frac{\partial a}{\partial \varphi}\right)^{2} \\
& +\frac{6 f^{2}}{a^{2}}\left(\frac{\partial a}{\partial \theta}\right)^{2}+\frac{4 f^{2}}{a} \frac{\partial^{2} a}{\partial \theta^{2}}=-\kappa_{6}^{2} b^{2} f^{2} \Lambda_{B}, \\
G_{05}= & \frac{4 \dot{b}}{a b} \frac{\partial a}{\partial \theta}+\frac{3 \dot{a}}{a^{2}} \frac{\partial a}{\partial \theta}-\frac{3}{a} \frac{\partial^{2} a}{\partial t \partial \theta}=0, \\
G_{06}= & \frac{4 \dot{b}}{a b} \frac{\partial a}{\partial \varphi}+\frac{3 \dot{a}}{a^{2}} \frac{\partial a}{\partial \varphi}-\frac{3}{a} \frac{\partial^{2} a}{\partial t \partial \varphi}=0, \\
G_{56}= & \frac{4 f^{\prime}}{a f} \frac{\partial a}{\partial \varphi}-\frac{4}{a} \frac{\partial^{2} a}{\partial \theta \partial \varphi}=0 .
\end{aligned}
$$

As we mentioned above, both extra dimensions are initially noncompact. Here, we choose to compactify along the $\varphi$ coordinate by introducing two 4-branes at the points $\varphi$ $=\varphi_{1}$ and $\varphi=\varphi_{2}$ (and impose periodic boundary conditions). The brane energy-momentum tensors can be written as

$$
T_{N}^{M(i)}=\frac{\delta\left(\varphi-\varphi_{i}\right)}{b f^{2}} \operatorname{diag}\left(-\Lambda_{i},-\Lambda_{i},-\Lambda_{i},-\Lambda_{i},-\tilde{\Lambda}_{i}, 0\right)
$$

where $i=1,2$ denotes the two branes. Note that the requirement of satisfying 6D energy-momentum conservation, $T_{N ; M}^{M(i)}=0$, together with the jump conditions (discussed shortly) forces one to introduce (1) an "inhomogeneity" reflected in the prefactor of Eq. (2.10) and (2) an "anisotropy" such that $T_{5}^{5(i)} \neq T_{k}^{k(i)}$ for $k=0, \ldots, 3$. On the other hand, as expected, the energy-momentum tensor in the bulk is dominated by the presence of a smoothly distributed bulk cosmological constant: i.e.,

$$
T_{N}^{M(B)}=\operatorname{diag}\left(-\Lambda_{B},-\Lambda_{B},-\Lambda_{B},-\Lambda_{B},-\Lambda_{B},-\Lambda_{B}\right),
$$

whose sign remains arbitrary at this point. Note that, in the absence of a radion potential, we do not include an additional contribution to the (55) or (66) component here.

Under the assumption that the two 4-branes are infinitely thin, the discontinuity in the first derivative of the metric tensor along the $\varphi$ coordinate creates a $\delta$-function contribution to its second derivative. The jump conditions [16] that follow by matching the coefficients of the $\delta$ functions on both sides of Einstein's equations provide constraints on the first derivatives of the warp factor at the location of the branes. One may easily conclude, from the absence of any $\delta$-function source on the right-hand side of the (66) component of Einstein's equations, Eq. (2.6), that there is no discontinuity in the first derivative of the warp factor along the $\theta$ coordinate due to the smooth distribution of energy along the longitudinal extra dimension. Then, from Eqs. (2.3) and (2.5), which contain second derivatives with respect to $\varphi$, we obtain, respectively, the constraints

$$
\left.\frac{\left[\partial_{\varphi} a\right]}{b a}\right|_{\varphi=\varphi_{i}}=-\frac{\kappa_{6}^{2}}{3} \Lambda_{i},\left.\quad \frac{\left[\partial_{\varphi} a\right]}{b a}\right|_{\varphi=\varphi_{i}}=-\frac{\kappa_{6}^{2}}{4} \tilde{\Lambda}_{i} .
$$

From the above conditions, it is obvious that the (ii) and (55) components of the energy-momentum tensor of each brane must satisfy the relation: $\widetilde{\Lambda}_{i}=4 \Lambda_{i} / 3$. Thus the brane tension along the "extra" $\theta$ coordinate is clearly distinguished from the remaining four, as pointed out below Eq. (2.10). Similar "anisotropic" brane tensions can be found elsewhere [5] in higher dimensional models.

As noted above, the inhomogeneous prefactor in Eq. (2.10) comes from the energy-momentum conservation constraint on $T_{N}^{M(i)}$. Let us assume for the moment that $T_{N}^{M(i)}$ is of the form $\left[g(\theta) \delta\left(\varphi-\varphi_{i}\right) / b\right] \operatorname{diag}\left(-\Lambda_{i},-\Lambda_{i},-\Lambda_{i}\right.$, $\left.-\Lambda_{i},-\tilde{\Lambda}_{i}, 0\right)$. We have allowed $g$ to be arbitrary and we have retained the common normalization $(1 / b)$. The $N=0$ conservation equation is identically zero for all solutions with $\dot{b}=0$, which includes all of the solutions we consider. Note that this constraint on $b$ stems from the anisotropy $\Lambda_{i}$ $\neq \tilde{\Lambda}_{i}$. The $N=1,2,3$ equations are trivial. The $N=5$ equation reduces to

$$
-\left(g^{\prime}+4 \frac{a^{\prime}}{a}+\frac{f^{\prime}}{f}\right) \tilde{\Lambda}_{i}+4 \frac{a^{\prime}}{a} \Lambda_{i}=0,
$$

with all derivatives taken with respect to $\theta$. With the anisotropy relation derived from the jump conditions this becomes

$$
\left(g^{\prime}+\frac{a^{\prime}}{a}+\frac{f^{\prime}}{f}\right) \tilde{\Lambda}_{i}=0 .
$$

As we will show in the next section, our solution further requires $a^{\prime} / a=f^{\prime} / f$, from which it can be easily seen that $g=1 / f^{2}$. We also note that without this tension prefactor the jump conditions would contain an explicit factor of $f(\theta)$ necessitating a constant $f$ and hence a flat internal space.

\section{STATIC 6D SOLUTIONS}

In this section we focus on the derivation of an exact 6-dimensional static solution with an exponential warp factor and a constant scale factor, $b(t)=b_{0}$. In this case, the nonvanishing components of Einstein's equations (after dropping all terms with time derivatives) take the form

$$
\begin{aligned}
& \frac{f^{\prime \prime}}{f}+\frac{3}{a^{2} f^{2}}\left(\frac{\partial a}{\partial \varphi}\right)^{2}+\frac{3}{a f^{2}} \frac{\partial^{2} a}{\partial \varphi^{2}}+\frac{3 f^{\prime}}{a f} \frac{\partial a}{\partial \theta}+\frac{3}{a^{2}}\left(\frac{\partial a}{\partial \theta}\right)^{2}+\frac{3}{a} \frac{\partial^{2} a}{\partial \theta^{2}} \\
& =-\kappa_{6}^{2} b_{0}^{2} \Lambda_{B}, \\
& \frac{6}{a^{2} f^{2}}\left(\frac{\partial a}{\partial \varphi}\right)^{2}+\frac{4}{a f^{2}} \frac{\partial^{2} a}{\partial \varphi^{2}}+\frac{4 f^{\prime}}{a f} \frac{\partial a}{\partial \theta}+\frac{6}{a^{2}}\left(\frac{\partial a}{\partial \theta}\right)^{2}=-\kappa_{6}^{2} b_{0}^{2} \Lambda_{B},
\end{aligned}
$$




$$
\begin{gathered}
\frac{6}{a^{2} f^{2}}\left(\frac{\partial a}{\partial \varphi}\right)^{2}+\frac{6}{a^{2}}\left(\frac{\partial a}{\partial \theta}\right)^{2}+\frac{4}{a} \frac{\partial^{2} a}{\partial \theta^{2}}=-\kappa_{6}^{2} b_{0}^{2} \Lambda_{B} \\
\frac{4 f^{\prime}}{a f} \frac{\partial a}{\partial \varphi}-\frac{4}{a} \frac{\partial^{2} a}{\partial \theta \partial \varphi}=0 .
\end{gathered}
$$

We assume that the dependence of the warp factor on the two extra coordinates can be written in a factorized form: $a(\theta, \varphi)=\Theta(\theta) \Phi(\varphi)$. Equation (3.4), then, provides a very strong constraint on the functions $\Theta(\theta)$ and $f(\theta)$ leading to the relation $\Theta(\theta)=A_{\theta} f(\theta)$, where $A_{\theta}$ is a constant. The difference of Eqs. (3.2) and (3.3), in turn, leads to the result

$$
\frac{\Phi^{\prime \prime}}{\Phi}=f f^{\prime \prime}-f^{\prime 2}=\omega^{2}
$$

where $\omega$ is again an arbitrary constant. The above equation allows us to write the general solution for $\Phi(\varphi)$ as a linear combination of increasing and decreasing exponentials. However, Eq. (3.3), which may be written as

$$
6\left(\frac{\Phi^{\prime}}{\Phi}\right)^{2}+6 f^{\prime 2}+4 f f^{\prime \prime}=-\kappa_{2}^{2} b_{0}^{2} f^{2} \Lambda_{B},
$$

restricts the form of the solution by demanding $\Phi^{\prime} / \Phi$ to be a constant as well. As a result, we may write the solution for $\Phi$ in the form

$$
\Phi(\varphi)=A_{\varphi} e^{ \pm \omega \varphi}
$$

where $A_{\varphi}$ is an integration constant. Equation (3.7), together with Eqs. (3.5) and (3.6), brings the equation for the remaining unknown metric function, $f(\theta)$, to the form

$$
f^{\prime 2}-\lambda^{2} f^{2}+\omega^{2}=0
$$

whose general solution can be written as

$$
f(\theta)=\frac{1}{4 \lambda}\left[e^{ \pm \lambda\left(\theta-\theta_{0}\right)}+4 \omega^{2} e^{\mp \lambda\left(\theta-\theta_{0}\right)}\right],
$$

where

$$
\lambda^{2}=-\frac{\kappa_{6}^{2} b_{0}^{2}}{10} \Lambda_{B}
$$

Note that the parameter $\lambda$ can take real or imaginary values for a negative or positive, respectively, bulk cosmological constant. We will comment on these two different options shortly.

We can furthermore easily check that the combination of Eqs. (3.5) and (3.6) trivially satisfies the remaining equation (3.1). We may, therefore, write the full solution for the warp factor, that multiplies the line-element of the 4-dimensional spacetime, as

$$
a(\theta, \varphi)=\frac{a_{0}}{4 \lambda} e^{ \pm \omega \varphi}\left[e^{ \pm \lambda\left(\theta-\theta_{0}\right)}+4 \omega^{2} e^{\mp \lambda\left(\theta-\theta_{0}\right)}\right]
$$

where $a_{0}$ stands for the product $A_{\varphi} A_{\theta}$. For simplicity, we may choose to place one 4-brane at the point $\varphi=0$ and the second one at $\varphi=L$. Then, by imposing the condition $a(\theta$ $\left.=\theta_{0}, \varphi=0\right)=1$, the integration constant $a_{0}$ may be fixed in terms of $\lambda$ and $\omega$.

Clearly, the $\varphi$ coordinate corresponds to a noncompact extra dimension as the conformal factor increases, or decreases, exponentially. The similarity with the extra fifth dimension of the Randall-Sundrum model is striking: the size of this dimension can become finite only by introducing two branes (3-branes in the case of the RS1 model, 4-branes in this case) at two different points along the transverse $\varphi$ dimension. Then, the interbrane distance defines the size of the extra dimension. The monotonic behavior of the scale factor in the $\varphi$ direction calls for the introduction of a pair of branes with positive and negative tension, as discussed earlier. We will show that this is indeed the case at the end of this section. Single-brane configurations could be also accommodated in our analysis by sending the second brane to an infinite distance from the first one.

At this point we can easily derive the relation between the internal 2D curvature and the bulk cosmological constant. The 2-dimensional curvature scalar is

$$
R_{(2)}=R_{5}^{5}+R_{6}^{6}=-\frac{2}{b_{0}^{2}} \frac{f^{\prime \prime}}{f} .
$$

From the solution (3.9) we further see that $f^{\prime \prime} / f=\lambda^{2}$, so that

$$
R_{(2)}=\frac{\kappa_{6}^{2}}{5} \Lambda_{B} .
$$

The behavior of the warp factor along the $\theta$ dimension, and subsequently the topology of this dimension, is strictly defined by the sign of the $\lambda^{2}$ parameter which determines the sign of the two-space curvature through Eq. (3.12), or equivalently through Eq. (3.10), by the sign of the bulk cosmological constant. We now distinguish the two cases:

$$
\text { A. } \boldsymbol{\lambda}^{2}>0
$$

This case corresponds, through Eq. (3.10), to a negative bulk cosmological constant and to a negatively curved twodimensional extra space time

$$
\Lambda_{B}<0, \quad R_{(2)}<0 .
$$

From the expression (3.9) we see that the function $f(\theta)$ is characterized by the existence of a minimum at

$$
\theta_{\min }=\theta_{0} \pm \frac{1}{\lambda} \ln 2 \omega
$$

under the assumption that $f(\theta)$ is symmetric under the transformation $\theta \leftrightarrow-\theta$. Identifying the two minima (for simplicity, we may set $\theta_{0}=0$ ), we can compactify this extra dimen- 
sion. Then, the quantity $2 \lambda \theta_{\min }$ is the physical size of the extra dimension, $2\left(b_{0} \theta_{\text {min }}\right)$, in units of $M_{6}^{-1}$.

If we allow the two symmetric branches of the function $f(\theta)$ to meet at $\theta=0$, a cusp is inevitably created. One might be tempted to introduce an infinitely thin 3-brane at this point creating a setup that resembles the $5 \mathrm{D}$ single-brane configurations presented in Ref. [7]. However, the absence of any discontinuity in the first and second derivative of the warp factor with respect to the $\theta$ coordinate does not allow for the introduction of any infinitely thin 3-brane in the model. The only allowed configuration is the one where each one of the 4-branes defining the size of the $\varphi$ dimension is a thick 3-brane extending along the $\theta$ dimension similar to the one described in Refs. [7,17]. In that case, the warp factor and its derivatives with respect to the $\theta$ coordinate are everywhere well defined as demanded. Introducing a thick 3-brane along the $\theta$ dimension will not spoil the solution (3.11) found above: it would merely render it as the solution outside the "wall" of the 3-brane where the minimum must take place.

\section{B. $\lambda^{2}<0$}

This case arises under the assumption that the bulk cosmological constant takes a positive value. The internal curvature of the $(\varphi, \theta)$-submanifold, in this case, is also positive according to the definition (3.12). Since $\lambda^{2}<0$, we may write $\lambda=i \tilde{\lambda}$, where $\tilde{\lambda}$ is a real number. It turns out that for special values of the parameter $\omega$, the $\theta$-dependent part of the solution for the warp factor (3.11) becomes periodic, and thus spontaneously compactified without the need for the introduction of any thin or thick 3-branes. However, in each case the resulting form of the line element of the 6D spacetime contradicts basic assumptions of this analysis.

Thus, if we choose $\omega^{2}=1 / 4$, the solution for $f(\theta)$ is given in terms of a cos-type function. However, the presence of the coefficient $1 /(4 \lambda)$ in Eq. (3.9) renders this metric function imaginary leading to

$$
\begin{aligned}
d s^{2}= & a^{2}(\theta, \varphi)\left(-d t^{2}+d x^{2}+d y^{2}+d z^{2}\right) \\
& +b_{0}^{2}\left[d \theta^{2}-\widetilde{f}(\theta)^{2} d \varphi^{2}\right],
\end{aligned}
$$

where we have set $f(\theta)=i \widetilde{f}(\theta)$. As a result, the character of the $\varphi$ dimension changes from space-like to time-like which is in disagreement with our argument that the $\varphi$ dimension plays the role of the extra transverse dimension of the RS model. If, alternatively, we choose $\omega^{2}=-1 / 4$, the function $f(\theta)$ comes out to be proportional to a sin-type function, however, in this case, $\omega$ itself comes out to be imaginary. If we perform the following coordinate transformation

$$
\varphi \rightarrow 4 H \tilde{t}, \quad t \rightarrow \frac{i \tilde{\varphi}}{4 H}
$$

the $6 \mathrm{D}$ line element takes the form

$$
\begin{aligned}
d s^{2}= & \sin ^{2}\left[\lambda\left(\theta-\theta_{0}\right)\right]\left\{-d \tilde{t}^{2}+e^{2 H \tilde{t}}\left(d x^{2}+d y^{2}+d z^{2}\right.\right. \\
& \left.\left.+\frac{d \widetilde{\varphi}^{2}}{16 H^{2}}\right)\right\}+b_{0}^{2} d \theta^{2}
\end{aligned}
$$

where $H=\tilde{\lambda} /\left(2 b_{0}\right)$ and where, for simplicity, we have set $a_{0}=2 \tilde{\lambda}$ in Eq. (3.11). The above line element describes a 6-dimensional spacetime whose four spatial dimensions are inflating. This is clearly in contradiction with our main goal to derive solutions with static extra dimensions.

We finally turn to the jump conditions that will help us determine the $\omega$ parameter of the solution as well as the number of fine-tunings that the model demands. Substituting the solution for $\Phi(\varphi)$ [Eq. (3.7)] into the jump conditions (2.12), we obtain the result

$$
\pm \frac{3 \omega}{\kappa_{6}^{2} b_{0}}=-\Lambda_{1}=\Lambda_{2} .
$$

The same fine-tuning between the two brane tensions, that was necessary for the RS solution to be consistent with the boundary conditions, appears also in our model. Note, however, that the second RS fine-tuning, between each brane tension and the bulk cosmological constant, is absent. The value of $\Lambda_{B}$ defines the parameter $\lambda$ while $\omega$ is defined in terms of the brane tensions. No relation between $\lambda$ and $\omega$ exists in our model, and thus, $\Lambda_{B}$ and $\Lambda_{i}$ remain uncorrelated. Nevertheless, since only one of the two brane tensions has been fixed so far, another fundamental parameter of our model, if not the second brane tension or the bulk cosmological constant, should be fixed instead, in this case. Through Eq. (3.15) the physical size of the $\theta$ dimension is given in terms of the $\lambda$ and $\omega$ parameters. By using Eqs. (3.15) and (3.19) we obtain the result

$$
\frac{3 e^{\lambda \theta_{\min }}}{2 \kappa_{6}^{2} b_{0}}=\left|\Lambda_{i}\right|,
$$

where $\left|\Lambda_{i}\right|$ stands for the absolute value of the brane tensions. The above relation fixes the physical size of the longitudinal extra dimension, which constitutes a fundamental parameter of the model, in terms of the brane tensions. The logarithmic dependence on the value of $\left|\Lambda_{i}\right|$ ensures the smallness of the size of the longitudinal dimension even for large values of the brane tension. It is therefore the existence of this extra dimension that introduces an additional parameter in the model whose fixing replaces the fine-tuning between bulk and brane parameters. Note, however, that the locations of the two 4-branes along the transverse $\varphi$ dimension and, thus, the size of this dimension remain a free parameter. We address this point in the next section.

A final comment on the number of fine-tunings is in order at this point: had the solutions for $\lambda^{2}<0$ led to a consistent spontaneous compactification of the $\theta$ dimension, an additional problem would appear: the special values of the $\omega$ parameter, for which these solutions arise, would result in the 
fixing of the values of both brane tensions through Eq. (3.19). The fine-tuning between brane tensions and bulk cosmological constant would still be absent, however, the number of necessary fine-tunings in the model would be again two. We might therefore conclude that periodic solutions, which respect the assumptions of our model and, at the same time, demand less fine-tuning than the usual 5D brane models, cannot arise in the framework of our analysis.

\section{NONSTATIC 4D SOLUTIONS}

Next, we proceed to construct 6-dimensional solutions with a 4-dimensional time-dependent submanifold but with a constant radion field once again. The source of this time dependence will be a nonvanishing 4D effective cosmological constant. Solutions similar to these but in the presence of only one extra dimension have been derived before [18]. Here, we will investigate the possibility of whether such solutions arise in the case of one additional, extra, longitudinal dimension. As in the previous section, we are going to assume that, initially, both extra dimensions are noncompact with the size of the $\varphi$ dimension becoming finite due to the presence of the two 4-branes while the $\theta$ dimension will be appropriately warped and thus spontaneously compactified.

Going back to the full time-dependent Einstein's equations (2.3)-(2.9), we try once again the factorized ansatz: $a(t, \theta, \varphi)=T(t) \Theta(\theta) \Phi(\varphi)$. The off-diagonal component (2.9) gives, as in the static case, a proportionality relation, $\Theta(\theta)=A_{\theta} f(\theta)$, between the two $\theta$-dependent functions of the metric tensor. The remaining off-diagonal equations, Eqs. (2.7) and (2.8), in conjunction with the above factorized ansatz, both lead to the result $b=$ const, that guarantees the staticity of the extra 2-dimensional space time.

Subtracting Eq. (2.6) from Eq. (2.5) we recover one of the two equations that determine the solutions for the metric functions $\Phi(\varphi)$ and $f(\theta)$, namely

$$
\frac{\Phi^{\prime \prime}}{\Phi}=f f^{\prime \prime}-f^{\prime 2}=\omega^{2} .
$$

Before trying to derive the second equation, we need to determine the solution for the time-dependent function $T(t)$. Taking the sum of Eqs. (2.3) and (2.4) we find the result

$$
\frac{\dot{T}}{T}\left(\frac{2 \dot{T}}{T}-\frac{\ddot{T}}{\dot{T}}\right)=0 .
$$

Obviously, one solution of the above equation is the trivial one, $\dot{T}=0$, which leads to the static case of the previous section with the Minkowski-like 4D submanifold. Clearly, the model accepts another solution that may be determined by demanding the expression inside the brackets to be zero. Then, we obtain the alternative solution

$$
T(t)=\frac{1}{c_{0}\left(t-t_{0}\right)}
$$

where $c_{0}$ and $t_{0}$ are integration constants. If we pass from the conformal time $t$ to the physical time $\tilde{t}$, through the transformation $d \tilde{t}=T(t) d t$, we might easily see that the solution takes the form

$$
T(\tilde{t})=e^{H\left(\tilde{t}-\tilde{t}_{0}\right)} .
$$

In the above expression, $H=-c_{0}$ is again an integration constant, which may be either real or imaginary describing a de Sitter

$$
d s_{4}^{2}=-d \tilde{t}^{2}+e^{2 H \tilde{t}}\left(d x^{2}+d y^{2}+d z^{2}\right)
$$

or anti-de Sitter

$$
d s_{4}^{2}=d x^{2}+e^{2 H x}\left(-d \tilde{t}^{2}+d y^{2}+d z^{2}\right)
$$

4D submanifold, respectively. Since the Einstein's equations are all expressed in terms of the conformal time, we will continue using $t$, instead of $\tilde{t}$, and the form of the solution (4.3), instead of Eq. (4.4), for convenience. In terms of the conformal time, a real or imaginary $c_{0}$ will distinguish between a de Sitter or anti-de Sitter 4D submanifold.

Having determined the solution for $T(t)$, we now turn to Eq. (2.6), which can be brought to the form

$$
6\left(\frac{\Phi^{\prime}}{\Phi}\right)^{2}-6 \frac{b^{2} c_{0}^{2}}{A_{\theta}^{2} \Phi^{2}}+6 f^{\prime 2}+4 f f^{\prime \prime}=-\kappa_{2}^{2} b^{2} f^{2} \Lambda_{B} .
$$

Comparing the above with Eq. (3.6) we may easily see that we can recover the equation (3.8) for $f(\theta)$, if $\Phi(\varphi)$ satisfies the following equation

$$
\left(\frac{\Phi^{\prime}}{\Phi}\right)^{2}-\frac{b^{2} c_{0}^{2}}{A_{\theta}^{2} \Phi^{2}}-\omega^{2}=0 .
$$

The solution for the $\varphi$-dependent part of the warp factor, now, takes the form

$$
\Phi(\varphi)=\frac{b c_{0}}{A_{\theta} \omega} \sinh \left[\omega\left|\varphi-\varphi_{0}\right|\right]
$$

for de Sitter spacetime, and

$$
\Phi(\varphi)=\frac{b \operatorname{Im}\left(c_{0}\right)}{A_{\theta} \omega} \cosh \left[\omega\left(\varphi-\varphi_{0}\right)\right]
$$

for anti-de Sitter spacetime.

Since the basic equation for the metric function $f(\theta)$ has remained unchanged, the general solution given by Eqs. (3.9) and (3.10) still remains the same. The subsequent discussion on the possible ways of compactifying the $\theta$ dimension, in the case of a negative or positive bulk cosmological constant, and the main conclusions drawn at the end of Sec. III still hold. On the other hand, the time-dependence of the warp factor has radically changed the solution for its $\varphi$-dependent part. Instead of the monotonic exponential dependence that prevailed in the static case, the time-dependent case may accommodate both sinh- and cosh-type solutions. Solutions 
similar to the above usually arise in the case where classical [19] or quantum [20] effects from bulk scalar fields are taken into account or when the effect of a bulk stabilizing potential is included in the model $[19,3]$. Note, however, that no such effects have been assumed to be present in our analysis. The conclusions drawn in the previous section from the jump conditions are also likely to change. Substituting the above solution for $\Phi(\varphi)$ into the jump conditions (2.12), we obtain the constraints

$$
\omega \operatorname{coth}\left[\omega\left(\varphi_{i}-\varphi_{0}\right)\right]=(-1)^{i} \frac{\kappa_{6}^{2}}{3} b_{0} \Lambda_{i}
$$

for the sinh-type solutions, and

$$
\omega \tanh \left[\omega\left(\varphi_{i}-\varphi_{0}\right)\right]=(-1)^{i} \frac{\kappa_{6}^{2}}{3} b_{0} \Lambda_{i}
$$

for the cosh-type solutions. In the above two equations, $i$ $=1,2$ denotes again the two 4-branes. The sinh-type solution with its monotonic behavior can clearly accommodate only pairs of positive-negative tension branes in analogy with the static case. Moreover, the specific solution is plagued by the existence of a singularity at the point $\varphi=\varphi_{0}$. In order to exclude the singularity from the $6 \mathrm{D}$ spacetime, both branes must be located on the same size of the singularity, i.e., $\varphi_{1}, \varphi_{2}<\varphi_{0}$. On the other hand, the cosh-type solution is everywhere well defined. The same point, $\varphi=\varphi_{0}$, corresponds, in this case, to a minimum which allows for pairs of positive tension branes to fit in the model. In both cases, either for sinh- or cosh-type solutions, the above jump conditions will fix the location of the two branes relative to the singularity or the minimum, respectively, in terms of the two brane tensions. Note, however, that the brane tensions, considered as input parameters of the model, remain totally uncorrelated. Moreover, the lack of fine-tuning between any of the brane tensions and the bulk cosmological constant still holds rendering the model free of any fine-tuning.

\section{RADION DYNAMICS}

The main goal of the previous sections was to find $6 \mathrm{D}$ solutions, static and nonstatic, with a constant radion field which demanded less or no fine-tuning of their parameters compared to other models in the literature. Nevertheless, an important aspect of these solutions needs to be studied next: do these solutions actually extremize the radion effective potential? And, if yes, is this extremum a minimum or a maximum of the effective potential?

In Ref. [10], a 5-dimensional "extremization" constraint that could serve as a consistency check for any solutions with a constant radion field was derived. It might be worth deriving the corresponding constraint in six dimensions and commenting on the possible differences that arise as one changes the number of extra dimensions. In order to do that we need to go back to the time-dependent Einstein's equations, and start by constructing the 4D trace of the energymomentum tensor [by taking the sum of Eq. (2.3) with three times Eq. (2.4)], which comes out to have the form

$$
\begin{aligned}
\kappa_{6}^{2} T_{\mu}^{\mu}= & -\frac{4 \dot{b}^{2}}{a^{2} b^{2}}-\frac{6 \ddot{b}}{a^{2} b}-\frac{12 \dot{a} \dot{b}}{a^{3} b}-\frac{6 \ddot{a}}{a^{3}}+\frac{4 f^{\prime \prime}}{b^{2} f}+\frac{12}{b^{2} f^{2} a^{2}}\left(\frac{\partial a}{\partial \varphi}\right)^{2} \\
& +\frac{12}{b^{2} f^{2} a} \frac{\partial^{2} a}{\partial \varphi^{2}}+\frac{12 f^{\prime}}{b^{2} f a} \frac{\partial a}{\partial \theta}+\frac{12}{b^{2} a^{2}}\left(\frac{\partial a}{\partial \theta}\right)^{2}+\frac{12}{b^{2} a} \frac{\partial^{2} a}{\partial \theta^{2}} .
\end{aligned}
$$

Next, we construct the following linear combination of all the components of the $6 \mathrm{D}$ energy-momentum tensor

$$
\begin{aligned}
\kappa_{6}^{2}\left[(4-n) T_{\mu}^{\mu}+3(n-2) T_{5}^{5}+3(n-2) T_{6}^{6}\right] \\
=-\left[(4-n) \frac{4 \dot{b}^{2}}{a^{2} b^{2}}+\frac{12 \ddot{b}}{a^{2} b}+\frac{24 \dot{a} \dot{b}}{a^{3} b}\right]-12(n-1) \frac{\ddot{a}}{a^{3}} \\
+(4-n) \frac{4 f^{\prime \prime}}{b^{2} f}+\frac{24}{b^{2}}\left[\frac{(n-1)}{f^{2} a^{2}}\left(\frac{\partial a}{\partial \varphi}\right)^{2}+\frac{1}{f^{2} a} \frac{\partial^{2} a}{\partial \varphi^{2}}\right. \\
\left.+\frac{f^{\prime}}{f a} \frac{\partial a}{\partial \theta}+\frac{(n-1)}{a^{2}}\left(\frac{\partial a}{\partial \theta}\right)^{2}+\frac{1}{a} \frac{\partial^{2} a}{\partial \theta^{2}}\right],
\end{aligned}
$$

where $n$ is an integer. In the case where the warp factor can be written as the product of two functions, one depending on the $4 \mathrm{D}$ coordinates and one on the extra coordinates, the above expression can be greatly simplified. If we write the warp factor in the factorized form

$$
a(t, \theta, \varphi)=T(t) W(\theta, \varphi),
$$

the expression in the last line of Eq. (5.2) may be written as

$$
\begin{aligned}
\frac{24}{b^{2}}[ & \frac{(n-1)}{f^{2} a^{2}}\left(\frac{\partial a}{\partial \varphi}\right)^{2}+\frac{1}{f^{2} a} \frac{\partial^{2} a}{\partial \varphi^{2}}+\frac{f^{\prime}}{f a} \frac{\partial a}{\partial \theta} \\
& \left.+\frac{(n-1)}{a^{2}}\left(\frac{\partial a}{\partial \theta}\right)^{2}+\frac{1}{a} \frac{\partial^{2} a}{\partial \theta^{2}}\right] \\
= & \frac{24}{n W^{n}} \frac{1}{\sqrt{g^{e x}}} \partial_{m}\left[\sqrt{g^{e x}} \partial^{m} W^{n}\left(x^{m}\right)\right]=\frac{24}{n W^{n}}\left(D_{m} D^{m} W^{n}\right),
\end{aligned}
$$

where $x^{m}$ denotes the extra coordinates $(\theta, \varphi)$ and $g_{m n}^{e x}$ is the metric tensor of the 2-dimensional extra spacetime. The above 2D double covariant derivative of the function $W^{n}$ divided by $W^{n}$ is the analog of the double derivative of the same function appearing in the 5D constraint of Ref. [10]. In the more general case where more than one, possibly nonflat, extra dimensions are present in the theory, the double derivative needs to be replaced with the covariant derivative in order for the internal geometry of the extra spacetime to be taken into account.

In addition, we may rewrite some of the other terms appearing in Eq. (5.2) in the following way

$$
12 \frac{\ddot{a}}{a^{3}}=\frac{2}{W^{2}} R_{(4)}, \quad \frac{4 f^{\prime \prime}}{b^{2} f}=-2 R_{(2)},
$$


where $R_{(4)}$ and $R_{(2)}$ stand for the $4 \mathrm{D}$ and $2 \mathrm{D}$, respectively, scalar curvature. Then, the constraint (5.2) takes the simplified form

$$
\begin{aligned}
\frac{24}{n W^{n}}\left(D_{m} D^{m} W^{n}\right) \\
=\kappa_{6}^{2}\left[(4-n) T_{\mu}^{\mu}+3(n-2) T_{5}^{5}+3(n-2) T_{6}^{6}\right] \\
+(n-1) \frac{2}{W^{2}} R_{(4)}+2(4-n) R_{(2)} \\
+\left[(4-n) \frac{4 \dot{b}^{2}}{a^{2} b^{2}}+\frac{12 \ddot{b}}{a^{2} b}+\frac{24 \dot{a} \dot{b}}{a^{3} b}\right] .
\end{aligned}
$$

Compared to the 5-dimensional one [10], the 6-dimensional version of the above constraint has a similar but more generalized structure. It involves all the extra components of the energy-momentum tensor, namely $T_{5}^{5}$ and $T_{6}^{6}$, as anticipated, and both on an equal footing. Moreover, the 2-dimensional scalar curvature of the extra spacetime explicitly makes its appearance together with the 4-dimensional one. Finally, the coefficients appearing in front of the 4D trace and extra components of the energy-momentum tensor seem to be "dimension" dependent. By mere comparison of the 5D and 6D versions of the constraint, we may easily conclude that the coefficient in front of the extra components behaves as (1 $+d)(n-2)$, where $d$ is the number of extra dimensions. However, the dependence of the coefficient of the 4D trace is more subtle and a higher dimensional calculation could only reveal its exact form in terms of $d$.

We would also like to stress here an additional point on the form of the constraint (5.6). It holds for every solution of the 6D Einstein's equations, even for the ones with a nonstatic extra spacetime. For the particular case of $n=1$ the above constraint can be interpreted as the equation of motion of the time-dependent scalar field $b(t)$. This can become clear if we rewrite Eq. (5.6) as

$$
\frac{2}{b^{2}} D_{\mu} D^{\mu} b^{2}=\kappa_{6}^{2}\left(T_{\mu}^{\mu}-T_{5}^{5}-T_{6}^{6}\right)+2 R_{(2)}-\frac{8}{W}\left(D_{m} D^{m} W\right) .
$$

For solutions with a nonstatic extra spacetime, the right-hand side of the above equation vanishes when an extremum, either minimum or maximum, is reached. For solutions with a constant radion field, which by definition corresponds to an extremum of the radion effective potential, the same combination should also vanish. Therefore the solutions found in the two previous sections, either static or nonstatic, but with constant $b$, should satisfy the above constraint. By using the following expressions for the components of the energymomentum tensor

$$
T_{\mu}^{\mu}=-4 \Lambda_{B}-\frac{4 \Lambda_{i}}{b_{0} f^{2}} \delta\left(\varphi-\varphi_{i}\right)
$$

$$
T_{5}^{5}=-\Lambda_{B}-\frac{\tilde{\Lambda}_{i}}{b_{0} f^{2}} \delta\left(\varphi-\varphi_{i}\right), \quad T_{6}^{6}=-\Lambda_{B}
$$

setting $W \equiv \Theta(\theta) \Phi(\varphi)$, and employing the form of the solutions found in Secs. III and IV, we obtain

$$
-2\left(\kappa_{6}^{2} \Lambda_{B}+10 \frac{\lambda^{2}}{b_{0}^{2}}\right)-\frac{8 \delta\left(\varphi-\varphi_{i}\right)}{b_{0} f^{2}}\left[\frac{\left[\partial_{\varphi} a\right]_{i}}{b_{0} a}+\frac{\kappa_{6}^{2}}{3} \Lambda_{i}\right]
$$

By using the jump conditions (2.12) and the definition of the $\lambda$ parameter from Eq. (3.10), we may easily conclude that both the static and nonstatic solutions derived in the previous sections satisfy the "extremization" constraint, as anticipated.

In order to answer the question whether the above extremum is a minimum or a maximum, a perturbation analysis around the above solutions needs to be performed, in which the time-dependent, small perturbation will be associated with the radion field. Therefore we consider the following ansatz for the line element of the six-dimensional spacetime

$$
\begin{aligned}
d s^{2}= & {\left[a_{0}^{2} f(\theta)^{2} \Phi(\varphi)^{2}+\epsilon A(\theta, \varphi) b(\widetilde{t})\right]\left(-d \widetilde{t}^{2}+e^{2 H \tilde{t}} d \vec{x}^{2}\right) } \\
& +[1+\epsilon B(\theta, \varphi) b(\widetilde{t})]\left(d \theta^{2}+f(\theta)^{2} d \varphi^{2}\right] .
\end{aligned}
$$

Note that we have switched to the system of nonconformal coordinates and we have set $b_{0}=1$ for simplicity. We have also chosen to perturb the de Sitter solutions found in the previous section; however, our analysis can be easily extended to the cases of Minkowski or anti-de Sitter branes by setting $H^{2} \rightarrow 0$ or $H^{2} \rightarrow-H^{2}$ (with the $\tilde{t}$-dependent perturbations replaced by $x$-dependent), respectively.

The above ansatz when substituted into the 6D Einstein's equations will lead to a system of differential equations and constraints that govern the behavior of the new line element. We will work in the linear order approximation and, thus, we are keeping only terms proportional to the small parameter $\epsilon \ll 1$. In this approximation, the generalized (00), (ii), (05), and (06) components of Einstein's equations take the form

$$
\begin{aligned}
(B & \left.+\frac{A}{a_{0}^{2} f^{2} \Phi^{2}}\right) 3 H \dot{b}-\left(\kappa_{6}^{2} a_{0}^{2} f^{2} \Phi^{2} \Lambda_{B}-3 H^{2}\right) B b-\frac{A b f^{\prime \prime}}{f} \\
& -\frac{3 b}{2 f^{2}} \frac{\partial^{2} A}{\partial \varphi^{2}}-\frac{3 b f^{\prime}}{2 f} \frac{\partial A}{\partial \theta}-\frac{3 b}{2} \frac{\partial^{2} A}{\partial \theta^{2}}-\frac{a_{0}^{2} b \Phi^{2}}{2} \\
& \times\left(\frac{\partial^{2} B}{\partial \varphi^{2}}+f f^{\prime} \frac{\partial B}{\partial \theta}+f^{2} \frac{\partial^{2} B}{\partial \theta^{2}}\right)=\kappa_{6}^{2} A b \Lambda_{B}
\end{aligned}
$$




$$
\begin{array}{cc}
\left(B+\frac{A}{a_{0}^{2} f^{2} \Phi^{2}}\right)(-2 H \dot{b}-\ddot{b})+\left(\kappa_{6}^{2} a_{0}^{2} f^{2} \Phi^{2} \Lambda_{B}-3 H^{2}\right) B b & \frac{\left(D_{\mu} D^{\mu} b\right)}{a_{0}^{2} f^{2} \Phi^{2}}-\frac{12 b}{f^{2}}\left(\frac{\Phi^{\prime 2}}{\Phi^{2}}-\frac{H^{2}}{a_{0}^{2} \Phi^{2}}\right)-\frac{12 b f^{\prime 2}}{f^{2}}-\frac{4 b \Phi^{\prime \prime}}{f^{2} \Phi} \\
+\frac{A b f^{\prime \prime}}{f}+\frac{3 b}{2 f^{2}} \frac{\partial^{2} A}{\partial \varphi^{2}}+\frac{3 b f^{\prime}}{2 f} \frac{\partial A}{\partial \theta}+\frac{3 b}{2} \frac{\partial^{2} A}{\partial \theta^{2}} & =\kappa_{6}^{2} b \Lambda_{B}, \\
+\frac{a_{0}^{2} b \Phi^{2}}{2}\left(\frac{\partial^{2} B}{\partial \varphi^{2}}+f f^{\prime} \frac{\partial B}{\partial \theta}+f^{2} \frac{\partial^{2} B}{\partial \theta^{2}}\right)=-\kappa_{6}^{2} A b \Lambda_{B}, & \frac{\left(D_{\mu} D^{\mu} b\right)}{a_{0}^{2} f^{2} \Phi^{2}}-\frac{b}{f^{2}}\left(\frac{8 \Phi^{\prime 2}}{\Phi^{2}}-\frac{12 H^{2}}{a_{0}^{2} \Phi^{2}}\right)-\frac{12 b f^{\prime 2}}{f^{2}}-\frac{4 b f^{\prime \prime}}{f} \\
=\kappa_{6}^{2} b \Lambda_{B},
\end{array}
$$$$
\left(2 B+\frac{3 A}{a_{0}^{2} f^{2} \Phi^{2}}\right) \frac{f^{\prime}}{f}-\frac{3}{2 a_{0}^{2} f^{2} \Phi^{2}} \frac{\partial A}{\partial \theta}-\frac{1}{2} \frac{\partial B}{\partial \theta}=0,
$$$$
\left(2 B+\frac{3 A}{a_{0}^{2} f^{2} \Phi^{2}}\right) \frac{\Phi^{\prime}}{\Phi}-\frac{3}{2 a_{0}^{2} f^{2} \Phi^{2}} \frac{\partial A}{\partial \varphi}-\frac{1}{2} \frac{\partial B}{\partial \varphi}=0,
$$

respectively.

By taking the sum of the (00) and (ii) components, we end up with the constraint

$$
\left(B+\frac{A}{a_{0}^{2} f^{2} \Phi^{2}}\right)(H \dot{b}-\ddot{b})=0,
$$

which demands that one of the two expressions inside the brackets vanishes. If we assume that the expression inside the first bracket is zero, then we can determine the exact form of the unknown functions $A(\theta, \varphi)$ and $B(\theta, \varphi)$ by plugging this constraint into the off-diagonal components (5.13) and (5.14). Then, we find that

$$
A(\theta, \varphi)=1, \quad B(\theta, \varphi)=-\frac{1}{a_{0}^{2} f(\theta)^{2} \Phi(\varphi)^{2}},
$$

modulo a multiplicative constant. Both of the equations (5.11) and (5.12), in that case, reduce to the background equation (4.8) (with $c_{0}^{2}$ being replaced by $H^{2}$ due to the use of nonconformal coordinates) which is obviously satisfied by the background solution. However, the solution (5.16) fails to satisfy any of the remaining components of Einstein's equations. For example, the off-diagonal (56) component, which has the form

$$
\begin{aligned}
\frac{2 f^{\prime}}{f}\left(\frac{\partial A}{\partial \varphi}-A \frac{\Phi^{\prime}}{\Phi}\right)+a_{0}^{2} f^{2} \Phi^{2}\left(\frac{f^{\prime}}{f} \frac{\partial B}{\partial \varphi}+\frac{\Phi^{\prime}}{\Phi} \frac{\partial B}{\partial \theta}\right)+\frac{\Phi^{\prime}}{\Phi} \frac{\partial A}{\partial \theta} \\
-\frac{\partial^{2} A}{\partial \theta \partial \varphi}=0
\end{aligned}
$$

leads to the constraint $f^{\prime} \Phi^{\prime}=0$ which is obviously in contradiction with the form of the background solution. Finally the (55) and (66) components, which after using Eq. (5.16) take the simplified forms

respectively, also turn out to lead to an inconsistent result. As a result, the option that the first expression inside the brackets in Eq. (5.15) is zero needs to be excluded. We are therefore left with the alternative option $(H \dot{b}-\ddot{b})=0$ which leads to an exponential solution of the time-dependent perturbation. By employing the equation of motion of the "radion" field

$$
g^{\mu \nu} D_{\mu} D_{\nu} b=m^{2} b
$$

and the exponential form of its solution, we get the result $m^{2}=-4 H^{2}$. Obviously, the mass squared of the radion field turns out to be negative for de Sitter 4D subspace, zero for Minkowski, and positive for anti-de Sitter 4D subspace.

The above result is in perfect agreement with similar works conducted in five dimensions: in Refs. [9-11] (see also [12]), it has been shown that the system of two Minkowski branes with a zero total brane tension cannot be stabilized since it leads to a radion field with a zero mass. In that case, the extremization of the radion potential follows from the fine-tuning of the parameters of the model which, however, cannot create a unique minimum for the radion field in the absence of a physical stabilization mechanism. As a result, the static solution derived in Sec. II describing a pair of flat branes cannot be stabilized and the radion field remains always massless. The same conclusion was drawn in Refs. $[13,14]$ where the stability of curved branes was also studied. According to their results, a pair of de Sitter branes leads to an effective theory for the radion field with a negative mass squared while a pair of anti-de Sitter branes turns out to be stable since it leads to a positive radion mass squared. Therefore our solution (4.9) corresponding to two branes with a positive effective cosmological constant is unstable under small time-dependent perturbations while the alternative solution described by (4.10) and corresponding to two branes with negative effective cosmological constant is stable. We bear in mind that this conclusion ignores any additional contribution to the $4 \mathrm{D}$ radion potential as might be expected from a more complete model which includes supersymmetry and supersymmetry breaking. Let us finally note that the above results are also in agreement with those of Ref. [6] where the extremum of the radion effective potential was a global minimum only in the case of a $4 \mathrm{D}$ negative cosmological constant. 


\section{CONCLUSIONS}

In this paper we have presented a 6D brane world model in the framework of which we addressed a number of important issues that arise in the context of higher-dimensional brane models. The relaxation of the fine-tuning of the fundamental parameters of the model was one of them: solutions with less or no fine-tuning at all, compared to their 5D ana$\log$ s, were constructed where the severe correlation between brane and bulk parameters was replaced by the fixing of the sizes of the two extra dimensions. In this way, another problem, that of the determination of the interbrane distance, was automatically resolved. The solutions derived allow for the introduction of pairs with positive-negative brane tensions, in analogy with the RS model, however, more physically interesting configurations with pairs of only positive tension branes were also found. Finally, the issue of the behavior of the aforementioned solutions, under time-dependent perturbations around configurations with a constant radion field, was examined and conclusions regarding their stability were derived.

In more detail, considering a 2D internal space of constant curvature, an exact static solution was first presented. The warp factor exhibits neither spherical nor cylindrical symmetry but depends on both extra coordinates. Two 4-branes are introduced at two different points along the so-called "transverse" extra dimension, along which the warp factor is a pure exponential resembling the profile of the warp factor in the case of the 5D RS model. The remaining extra dimension, the "longitudinal" one, along which the 4-branes extend, was shown to be "spontaneously" compactified in the case of a negative bulk cosmological constant, or equivalently of a negatively curved internal space. Although the consistency of the bulk solution with the brane boundary conditions demand the two branes to have exactly equal and opposite tensions, no correlation exists between the brane tensions and the bulk cosmological constant. Instead, it is the size of the longitudinal extra dimension that is fixed through the jump conditions in terms of the value of the brane tensions.

The above solution, although it is characterized by reduced fine-tuning of its fundamental parameters, has the interbrane distance along the transverse extra dimension as a free parameter. In an attempt to resolve this problem, too, we then derived a nonstatic solution whose 4D subspace corresponds to a de Sitter or anti-de Sitter spacetime. These solutions have a number of positive features: first, being non- static due to the presence of an effective 4D cosmological constant, the only fine-tuning of the model, the one between the two brane tensions, is also relaxed rendering the model free of any fine-tuning; second, although they have the same profile along the compactified, longitudinal dimension, the exponential behavior along the transverse one changes to a cosh- or sinh-type one, a fact which has two important consequences: the fixing of the locations of the two branes and, thus, of the interbrane distance, and the accommodation of pairs of positive tensions branes instead of only pairs of positive-negative tensions.

The fixing of the physical interbrane distance and thus of the size of the extra dimension relies on the assumption that the scale factor along the internal spacetime remains constant. Both of the above solutions, static or nonstatic, were derived under this assumption, or, alternatively, that the corresponding "radion" field was already at the extremum of its effective potential. An "extremization" constraint, valid for solutions with a static or nonstatic radion field whose effective potential possess an extremum, was formulated and used as a consistency check for our solutions. The final issue of whether this extremum was a unique minimum or merely a local maximum of the radion effective potential needed to be addressed. The background solutions with a constant scale factor along the extra dimensions were perturbed and a linear stability analysis was performed. Our analysis revealed the stability of the nonstatic solutions describing an anti-de Sitter 4D spacetime and accommodating pairs of only positive tension branes. The remaining solutions describing 4D de Sitter and Minkowski spacetimes were found to correspond to local maxima and saddle points, respectively, in close analogy to similar analyses performed in five dimensions.

\section{ACKNOWLEDGMENTS}

We are deeply grateful to Luigi Pilo, Maxim Pospelov, and Alexander Zhuk for useful discussions. P.K. would also like to acknowledge financial support by the DOE Grant No. DE-FG-02-94-ER-40823 and by the EC TMR Contract No. HRPN-CT-2000-00148 during the early and late stages of this work, respectively, as well as the CERN Theory Division for the hospitality and financial support while this work was in progress. R.M. wishes to thank the CERN Theory Division for its hospitality during a portion of this work. The work of K.O. was supported in part by DOE Grant No. DEFG-02-94-ER-40823 at the University of Minnesota.
[1] L. Randall and R. Sundrum, Phys. Rev. Lett. 83, 3370 (1999).

[2] M. Gogberashvili, hep-ph/9812296; T. Li, Phys. Lett. B 471, 20 (1999); J. Lykken and L. Randall, J. High Energy Phys. 06, 014 (2000); I. Oda, Phys. Lett. B 472, 59 (2000); 480, 305 (2000); G. K. Leontaris and N. E. Mavromatos, Phys. Rev. D 61, 124004 (2000); 64, 024008 (2001); V. Barger, T. Han, T. Li, J. D. Lykken, and D. Marfatia, Phys. Lett. B 488, 97 (2000); S. Ichinose, hep-th/0003275; Class. Quantum Grav. 18, 421 (2001); S. Mukohyama, Phys. Rev. D 63, 044008 (2001);
E. Flanagan, N. Jones, H. Stoica, S. H. Tye, and I. Wasserman, ibid. (to be published), hep-th/0012129; M. A. Luty and R. Sundrum, hep-th/0012158; I. Quiros, hep-th/0101030.

[3] P. Kanti, K. A. Olive, and M. Pospelov, Phys. Rev. D 62, 126004 (2000).

[4] A. G. Cohen and D. B. Kaplan, Phys. Lett. B 470, 52 (1999); A. Chodos and E. Poppitz, ibid. 471, 119 (1999); R. Gregory, Phys. Rev. Lett. 84, 2564 (2000); I. Olasagasti and A. Vilenkin, Phys. Rev. D 62, 044014 (2000); N. Arkani-Hamed, L. Hall, 
D. Smith, and N. Weiner, ibid. 62, 105002 (2000); T. Nihei, ibid. 62, 124017 (2000); M. Chaichian and A. B. Kobakhidze, Phys. Lett. B 478, 299 (2000); A. Chodos, E. Poppitz, and D. Tsimpis, Class. Quantum Grav. 17, 3865 (2000); T. Gherghetta, E. Roessl, and M. Shaposhnikov, Phys. Lett. B 491, 353 (2000); M. Gogberashvili and P. Midodashvili, hep-ph/0005298; S. Moon, S. Rey, and Y. Kim, Nucl. Phys. B602, 467 (2001); S. Corley and D. A. Lowe, Phys. Lett. B 505, 197 (2001); C. Charmousis, R. Emparan, and R. Gregory, J. High Energy Phys. 05, 026 (2001); O. Corradini and Z. Kakushadze, Phys. Lett. B 506, 167 (2001); S. Ichinose, hep-th/0103211; J. E. Kim, B. Kyae, and H. M. Lee, hep-th/0104150.

[5] Z. Chacko and A. E. Nelson, Phys. Rev. D 62, 085006 (2000); T. Gherghetta and M. Shaposhnikov, Phys. Rev. Lett. 85, 240 (2000); J. Chen, M. A. Luty, and E. Ponton, J. High Energy Phys. 09, 012 (2000); H. Collins and B. Holdom, Phys. Rev. D (to be published), hep-ph/0103103.

[6] U. Gunther and A. Zhuk, Phys. Rev. D 61, 124001 (2000).

[7] P. Kanti, I. I. Kogan, K. A. Olive, and M. Pospelov, Phys. Rev. D 61, 106004 (2000).
[8] C. Csáki, M. Graesser, L. Randall, and J. Terning, Phys. Rev. D 62, 045015 (2000).

[9] U. Gen and M. Sasaki, gr-qc/0011078.

[10] G. Gibbons, R. Kallosh, and A. Linde, J. High Energy Phys. 01, 022 (2001).

[11] A. Papazoglou, Phys. Lett. B 505, 231 (2001).

[12] T. Boehm, R. Durrer, and C. van de Bruck, Phys. Rev. D (to be published), hep-th/0102144.

[13] P. Binetruy, C. Deffayet, and D. Langlois, hep-th/0101234.

[14] Z. Chacko and P. J. Fox, Phys. Rev. D 64, 024015 (2001).

[15] R. M. Wald, General Relativity (University of Chicago, Chicago, 1984).

[16] W. Israel, Nuovo Cimento B 44, 1 (1966).

[17] P. Kanti, I. I. Kogan, K. A. Olive, and M. Pospelov, Phys. Lett. B 468, 31 (1999).

[18] N. Kaloper, Phys. Rev. D 60, 123506 (1999); H. B. Kim and H. D. Kim, ibid. 61, 064003 (2000).

[19] P. Kanti, K. A. Olive, and M. Pospelov, Phys. Lett. B 481, 386 (2000).

[20] R. Hofmann, P. Kanti, and M. Pospelov, Phys. Rev. D 63, 124020 (2001). 